\title{
Essential role of the cGMP/PKG signaling pathway in regulating the proliferation and survival of human renal carcinoma cells
}

\author{
YU REN $^{1 *}$, JIANJIAN ZHENG ${ }^{2 *}$, XUPING YAO ${ }^{1}$, GUOBIN WENG $^{3}$ and LING WU ${ }^{4}$ \\ ${ }^{1}$ Department of Urologic Surgery, Ningbo Urology and Nephrology Hospital, Ningbo, Zhejiang 315000; \\ ${ }^{2}$ Wenzhou Key Laboratory of Surgery, The First Affiliated Hospital of Wenzhou Medical College, Wenzhou, Zhejiang 325000; \\ ${ }^{3}$ Department of Urologic Surgery, Ningbo Yinzhou People's Hospital, ${ }^{4}$ Department of Pediatric Rheumatology \\ and Immunology, Ningbo Women and Children's Hospital, Ningbo, Zhejiang 315000, P.R. China
}

Received March 23, 2014; Accepted August 26, 2014

DOI: $10.3892 /$ ijmm.2014.1925

\begin{abstract}
Phosphodiesterase type 5 (PDE5) plays a key role in regulating the intracellular cyclic GMP (cGMP) concentration, which influences anti-proliferative and pro-apoptotic mechanisms in multiple carcinomas. PDE5 inhibitors, such as exisulind and its analogs have anticancer activities. In this study, we found that suppressing PDE5 gene expression by PDE5 siRNA inhibited cell proliferation and induced apoptosis in OS-RC-2 human renal cell carcinoma cells. These effects were enhanced by 8 -Br-cGMP, a cell membrane permeable cGMP derivative, and were inhibited by KT5823, a protein kinase $\mathrm{G}$ (PKG) inhibitor, indicating that PKG was activated by intracellular cyclic GMP. In addition, there was a reduction in both the mRNA and protein expression of cyclin D1, while p21 protein expression was increased; the reduction in cyclin D1 expression was blocked by the proteasome inhibitor, MG132, or c-Jun N-terminal kinase (JNK) inhibitor; both $\beta$-catenin and JNK were phosphorylated by activated PKG. Furthermore, p21 protein expression was decreased in Sp1 siRNA transfectedcells treated with 8 -Br-cGMP, indicating that $\mathrm{p} 21$ may be partly controlled by the PKG activation through Spl. Furthermore, we found that PKG I $\beta$ was responsible for the anticancer activities. Our findings indicate that the downregulation of PKG-activated genes, such as cyclin D1 partly accounts for the pro-apoptotic effects in PDE5 siRNA-transfected OS-RC-2 cells.
\end{abstract}

Correspondence to: Professor Xuping Yao, Department of Urologic Surgery, Ningbo Urology and Nephrology Hospital, 1 Qianhe Road, Yinzhou, Ningbo, Zhejiang 315000, P.R. China

E-mail: nbrenyu@163.com

Professor Guobin Weng, Department of Urologic Surgery, Ningbo Yinzhou People's Hospital, 251 Baizhang East Road, Ningbo, Zhejiang 315000, P.R. China

E-mail: dd_wgb@yahoo.com.cn

*Contributed equally

Key words: renal carcinoma cell, protein kinase G signaling, phosphodiesterase type 5 , apoptosis, $\beta$-catenin

\section{Introduction}

The incidence of kidney cancer has been rising steadily. Native Americans have the highest incidence of kidney cancer and highest mortality rates for males and females $(1,2)$. Renal cell carcinoma (RCC) is the most common type of kidney cancer in adults, responsible for approximately $80 \%$ of cases (3). At present, initial treatment is most commonly a radical or partial nephrectomy and remains the mainstay of curative treatment (1). RCC is resistant to chemotherapy and radiotherapy in most cases, but responds well to immunotherapy with interleukin-2 or interferon- $\alpha$ (2).

Phosphodiesterase 5 (PDE5) is a member of several cyclic nucleotide phoshodiesterase gene families, which hydrolyze cyclic GMP (cGMP-PDEs) (4). The abnormal expression of PDE5 and the increase in PDE5 activity has been detected in a variety of tumor cell lines (5-10). A sustained increase in intracellular cyclic GMP (cGMP) induced by the inhibition of PDE5 is required to modify the process of apoptosis and mitotic arrest in these carcinoma cells with an enhanced PDE5 expression $(5,6,8,9)$. The inhibition of PDE5 induces sustained levels of intracellular cGMP and activates its downstream protein kinase $\mathrm{G}(\mathrm{PKG})$, resulting in the inhibition of proliferation and the induction of apoptosis in colon cancer cells. Further studies exploring the mechanisms of the PDE5 inhibitorinduced activation of the downstream cGMP pathway found that the activated intracellular cGMP-PKG pathway increased the degradation of $\beta$-catenin in SW480 colon cancer cells, but not in SW620 cells $(11,12)$. In the present study, to determine the role of the PDE5 enzyme in controlling the inhibition and apoptosis of renal carcinoma cells, we suppressed PDE5 expression in OS-RC-2 human renal carcinoma cells by transfecting the cells with PDE5 siRNA, and investigated the changes in cell proliferation, apoptosis and the downstream molecules of PKG.

\section{Materials and methods}

MG132 (Z-leu-Leu-Leu-CHO) and S-Nitroso-N-acetylpenicillamine (SNAP) were purchased from Biomol Research Laboratories, Inc. (Plymouth Meeting, PA, USA) and Sigma (St. Louis, MO, USA), respectively. 8-Br-cGMP was obtained from Sigma. Anti- $\beta$-catenin, anti-phosphorylated (p)- $\beta$-catenin, 
anti-p-c-Jun N-terminal kinase (JNK), anti-cyclin A, anticyclin B1, KT5823, anti-PED5 and anti-PKG antibodies were purchased from Abcam (Cambridge, MA, USA). Anti-actin (AC-74), anti-cyclin D1 anti-p21, anti-p27 antibodies and JNK inhibitor (SP600125) were purchased from the Beyotime Institute of Biotechnology (Haimen, China). Anti-Spl antibody was purchased from Santa Cruz Biotechnology, Inc. (Santa Cruz, CA, USA).

Cell culture. The human renal carcinoma cell line, OS-RC-2, was obtained from the Type Culture Collection of the Chinese Academy of Sciences (Shanghai, China). The cells were cultured under $5 \% \mathrm{CO}_{2}$ at $37^{\circ} \mathrm{C}$ in RPMI-1640 medium with $5 \%$ FBS and $1 \%$ penicillin/streptomycin (all from HyClone, Auckland, New Zealand).

RNA interference. Target gene accession numbers NM_033437 and NM_003109 were obtained from GenBank. According to the principles of Ambion for the design of siRNAs, the sequences of 21 nucleotides beginning with AA and containing $30-50 \%$ GC were selected and used as target sites. The siRNA used were as follows: PDE5A siRNA, 5'-GGAAGAAACAAG AGAGCUAdTdT-3' (sense) and 5'-UAGCUCUCUUGUUUCU UCCdTdT-3' (antisense); Sp1 siRNA, 5'-CAUCCAAGGCUGU GGGAAAdTdT-3' (sense) and 5'-UUUCCCACAGCCUU GGAUG dTdT-3' (antisense). The control and negative control sequences were provide by the company, and a report was sent to ensure the effects. No homology sequence was found by Blast analysis. The siRNAs were purchased from Wuhan Genesil Biotechnology Co., Ltd. (Wuhan, China), and $10 \mathrm{nmol} / \mathrm{l}$ of each siRNA were transfected into the OS-RC-2 cells, using HiPerFect transfection reagent (Qiagen, Valencia, CA, USA).

Assessment of cell apoptosis and proliferation. The activity of caspase-3 was determined using the caspase-3 activity kit (Beyotime Institute of Biotechnology). Cell lysates were prepared after their respective treatment. Subsequently, the OS-RC-2 cells were scraped, centrifuged, resuspended and lysed in lysis buffer (RIPA buffer and protease inhibitor PMSF; Solarbio, Beijing, China). Assays were performed in 96-well microtiter plates by incubating $10 \mu \mathrm{l}$ protein of cell lysate per sample in $80 \mu \mathrm{l}$ reaction buffer (1\% NP-40, $20 \mathrm{mM}$ Tris- $\mathrm{HCl}$, $\mathrm{pH} 7.5,137 \mathrm{mM}$ Nad and $10 \%$ glycerol) containing $10 \mu \mathrm{l} /$ mM caspase-3 substrate (Ac-DEVD-pNA). The lysates were incubated at $37^{\circ} \mathrm{C}$ for $4 \mathrm{~h}$. Samples were measured using a microplate reader (Bio-Rad Laboratories, Inc., Hercules, CA, USA) at an absorbance of $405 \mathrm{~nm}$, according to the manufacturer's instructions. Cell proliferation was determined by MTT assay according to the instructions provided with the MTT cell proliferation assay kit (Beyotime Institute of Biotechnology). The absorbance was measured at $570 \mathrm{~nm}$ on a microplate reader (Bio-Rad Laboratories, Inc.).

Quantitative RT-PCR (RT-qPCR). Total RNA was extracted using TRIzol reagent (Invitrogen, Carlsbad, CA, USA), reverse-transcribed into cDNA templates and amplified using a ReverTra Ace qPCR RT kit (Toyobo, Osaka, Japan). RT-qPCR was performed using a SYBR-Green Real-time PCR Master Mix-Plus (Toyobo). The PCR primers (Table I) were used for gene amplification. Target gene expression levels are presented as a ratio of the levels in the treated cells and the control cells using the $\Delta \Delta \mathrm{Ct}$ method, as previously described (13).

Western blot analysis. The OS-RC-2 cells were collected using the rubber policeman, washed in phosphate-buffered saline (PBS) and lysed in ice-cold PBS buffer [0.1 M Tris- $\mathrm{HCl}$, $\mathrm{pH}$ 8.0, $100 \mathrm{mM} \mathrm{NaCl}, 1 \mathrm{mM}$ EDTA, 1\% Nonidet P-40, $0.5 \%$ sodium deoxycholate, $0.1 \%$ sodium dodecylsulfate (SDS), and $0.1 \%$ protease inhibitor cocktail and $10 \mathrm{mM}$ phenylmethylsulphonyl fluoride (PMSF)] for $30 \mathrm{~min}$ on ice. The lysates were sonicated for $2 \mathrm{sec}$ to shear the DNA to reduce its viscosity, followed by centrifugation at $28,100 \mathrm{x}$ g for $30 \mathrm{~min}$ at $4^{\circ} \mathrm{C}$. The supernatant was collected, and protein concentrations were measured using the Bradford method (Bio-Rad Laboratories, Inc.). Twenty micrograms of protein determined by Bradford assay were electrophoretically separated using a $12 \%$ sodium dodecyl sulfate-polyacrylamide gel electrophoresis (SDS-PAGE) gel and transferred onto polyvinylidene fluoride (PVDF) membranes, and then immunoblotted with the corresponding antibodies. Immuno-detection was performed with an enhanced chemiluminescence (ECL) detection kit (Beyotime Institute of Biotechnology). The protein bands were analyzed using Quantity One 4.4 software.

Construction and expression of PKG wild-type and mutants. The wild-type and mutant constructs of PKG, including PKG I $\alpha$ and PKG I $\beta$ were designed and purchased from BioTech Co., Ltd. The PKG constructs were subcloned into the expression vector pMIG upstream of the IRES-enhanced green fluorescent protein (GFP) sequence. The cells were transfected with pMIG as previously described (14).

Statistical analysis. All data are presented as the means \pm standard deviation (SD). Significant differences among the groups were determined using the unpaired Student's t-test. A value of $\mathrm{P}<0.05$ was accepted as an indication of statistical significance. All data in the figures were obtained from at least 3 independent experiments.

\section{Results}

PDE5 siRNA suppresses PDE5 expression at the posttranscriptional and protein level. We detected the mRNA and protein expression of PDE5 in the OS-RC-2 cells, and then used specific siRNA to silence PDE5 expression. Following transfection with PDE5 siRNA, real-time PCR revealed that the level of PDE5 mRNA expression was significantly reduced in the transfected cells when compared with the negative control (Fig. 1A). Western blot analysis also revealed a significant decrease in the levels of the $105 \mathrm{kDa}$ PDE5 in the OS-RC-2 cells transfected with PDE5 siRNA, when compared with the control (Fig. 1B and C). Thus, our data indicated that treatment with PDE5 siRNA successfully suppressed the expression of PDE5 in the OS-RC-2 cells.

PDE5 suppression reduces proliferation and increases apoptosis through cGMP-PKG. PDE5 siRNA-transfected OS-RC-2 cells had much lower growth rates than the control and negative control cells. The average rate of growth inhibition was approximately $50 \%$ in the PDE5 siRNA-transfected cells. 
Table I. Primer sequences for real-time RT-PCR.

\begin{tabular}{|c|c|c|}
\hline Genes & Primers sequences $\left(5^{\prime} \rightarrow 3^{\prime}\right)$ & Product length (bp) \\
\hline PDE5 & $\begin{array}{l}\text { Forward: CACACCGAATCTTGCTCTTG } \\
\text { Reverse: TCAGAATCCTTGACAACAATGG }\end{array}$ & 134 \\
\hline Cyclin D1 & $\begin{array}{l}\text { Forward: TCTACACCGACAACTCCATCCG } \\
\text { Reverse: TCTGGCATTTTGGAGAGGAAGTG }\end{array}$ & 133 \\
\hline $\mathrm{p} 21$ & $\begin{array}{l}\text { Forward: AGGTGGACCTGGAGACTCTCAG } \\
\text { Reverse: TCCTCTTGGAGAAGATCAGCCG }\end{array}$ & 94 \\
\hline PKG I $\alpha$ & $\begin{array}{l}\text { Forward: ACTCCACAAATGCCAGTCGG } \\
\text { Reverse: GGTGAACTTCCGGAATGCCT }\end{array}$ & 139 \\
\hline PKG I $\beta$ & $\begin{array}{l}\text { Forward: AGAGCGCGAGCACCTTG } \\
\text { Reverse: GATCTGCGACAGCTCCAAGT }\end{array}$ & 194 \\
\hline PKG II & $\begin{array}{l}\text { Forward: AAAAGACATGCGAAGCGGTC } \\
\text { Reverse: GCTCAACTCTTCCGAACCCA }\end{array}$ & 166 \\
\hline$\beta$-actin & $\begin{array}{l}\text { Forward: AATCGTGCGTGACATTAAG } \\
\text { Reverse: GAAGGAAGGCTGGAAGAG }\end{array}$ & 178 \\
\hline
\end{tabular}

PDE5, phosphodiesterase type; PKG, protein kinase G.

A

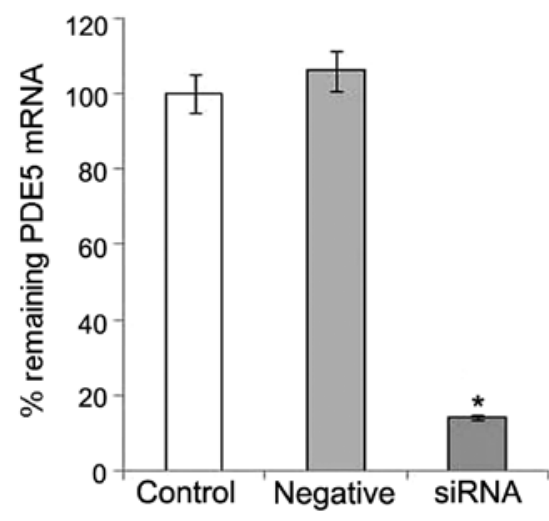

B
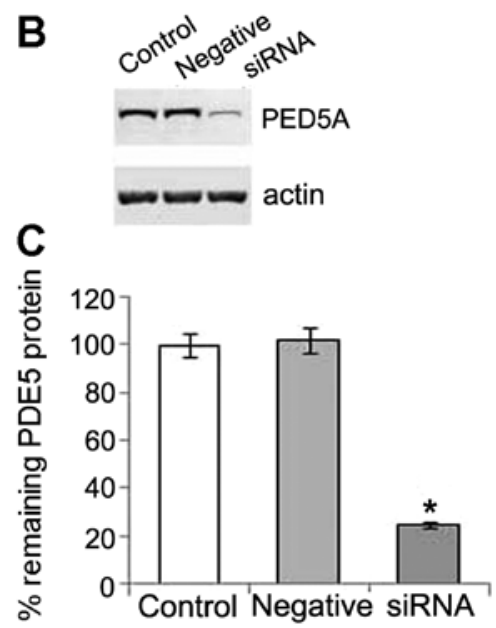

Figure 1. Effect of phosphodiesterase type 5 (PDE5) siRNA on the mRNA and protein expression of PDE5 in OS-RC-2 cells. PDE5 siRNA was transfected into OS-RC-2 cells for $48 \mathrm{~h}$. Compared with the control, PDE5 mRNA and protein expression was inhibited in the cells transfected with PDE5 siRNA. (A) PDE5 mRNA and (B) protein expression was determined by RT-qPCR and western blot analysis, respectively. (C) Percentage of PDE5 protein expression by western blot analysis. Data are presented as the means $\pm \operatorname{SD}(n=3) .{ }^{*} \mathrm{P}<0.05$.

When 8-Br-cGMP, a cell membrane permeable cGMP derivative was added, a further inhibition was observed. However, the inhibitory effect in the PDE5 siRNA-transfected OS-RC-2 cells was blocked by KT5823, an inhibitor of PKG (Fig. 2A). Conversely, there was a 2-fold increase in the relative level of caspase- 3 in the transfected cells compared to the control cells. Caspase- 3 is an early indicator of apoptosis. Caspase- 3 activity was enhanced 3-fold following treatment with 8-Br-cGMP and was inhibited following treatment with KT5823 (Fig. 2B).

Effect of PDE5 siRNA on the molecules related to cell cycle progression. To elucidate the mechanisms responsible for the reduced proliferation and increased apoptosis in the PDE5
siRNA-transfected cells, the levels of cell cycle-specific molecules, including cyclins (cyclin A, B and D1) and CDK inhibitors (CKIs, p21 and p27) were determined. Our results revealed that no changes in the levels of cyclin $\mathrm{A}$ and $\mathrm{B} 1$ were detected in the PDE5 siRNA-transfected cells; however, a $50 \%$ reduction in the levels of cyclin D1 was observed in these cells. Further study indicated that both the mRNA and protein expression of cyclin D1 was decreased in a time-dependent manner in these cells. A 2- to 3-fold increase in the expression of p21, a mitotic inhibitor, was observed in the PDE5 siRNAtransfected cells when compared to the control cells. However, there were no changes observed in p27 expression, another mitotic inhibitor involved in the cyclin D1-CDK2, CDK4 and 

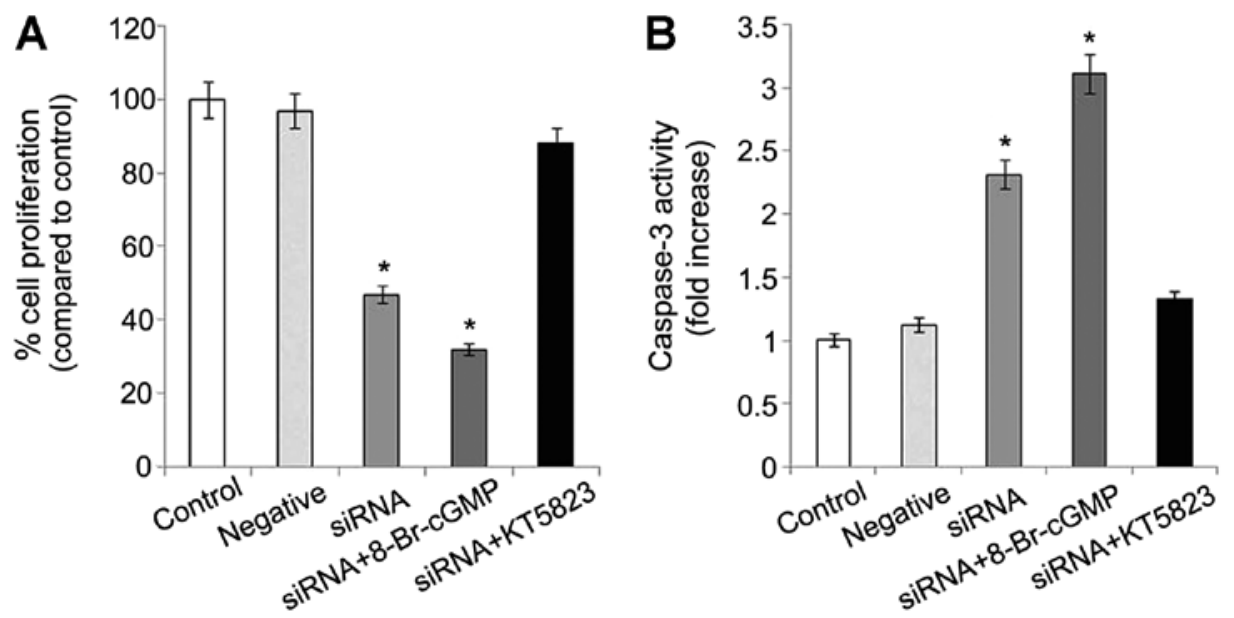

Figure 2. Response of OS-RC-2 cells to a combination of phosphodiesterase type 5 (PDE5) siRNA following 8-Br-cyclic GMP (cGMP) or KT5823 treatment in $24 \mathrm{~h}$. (A) Cell proliferation in OS-RC-2 cells following various treatments by MTT assay. (B) Caspase-3 activity in OS-RC-2 cells following various treatments . Data are presented as the means $\pm \mathrm{SD}(\mathrm{n}=3)$. ${ }^{*} \mathrm{P}<0.05$.

A

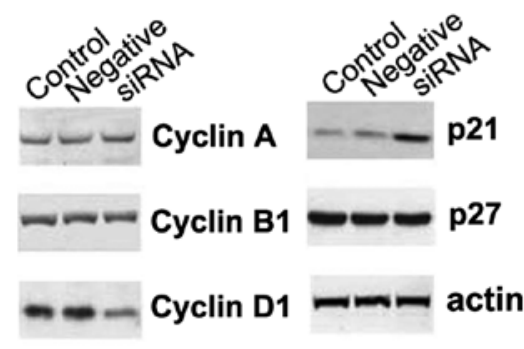

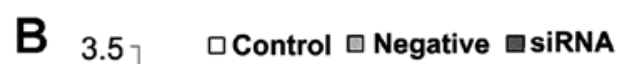

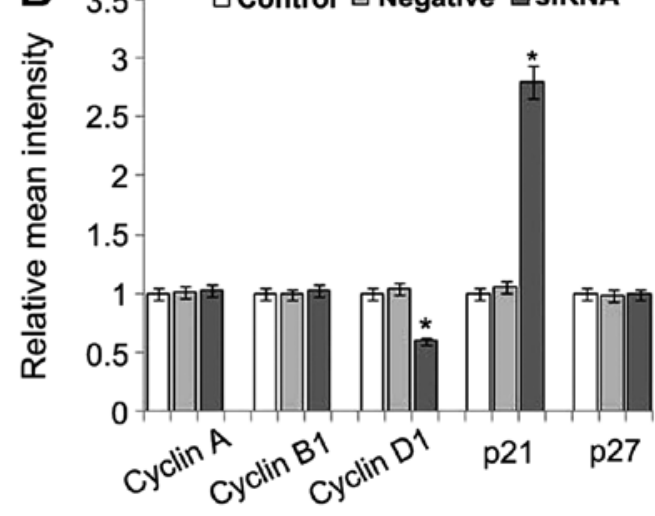

Figure 3. Effect of phosphodiesterase type 5 (PDE5) siRNA on the molecules related to cell cycle progression in OS-RC-2 cells. PDE5 siRNA was transfected into the OS-RC-2 cells for $24 \mathrm{~h}$. (A) Comparison of the expression of and mitotic inhibitors (p21 and p27), cyclins (A, B1 and D1) in siRNA-transfected OS-RC-2 cells and the control cells. (B) Changes in the expression of cyclins (A, B1 and D1) and mitotic inhibitors (p21 and p27) in siRNA-transfected OS-RC-2 cells and the control cells. Data are presented as the means \pm SD $(n=3)$. ${ }^{*} \mathrm{P}<0.05$.

cyclin E-CDK2 inhibition at the G1 phase (Fig. 3). Therefore, cyclin D1 and p21 may play an important role in the inhibition of proliferation and the increase in apoptosis.

Involvement of $\beta$-catenin/TCF and JNK pathways in regulation of cyclin D1 expression. To explore whether the time-dependent decrease in the expression of cyclin D1 involves the regulation of $\beta$-catenin in this study, we examined the mRNA and protein levels of $\beta$-catenin. Of note, the protein levels of $\beta$-catenin also showed a time-dependent reduction following transfection. However, no changes in the mRNA level of $\beta$-catenin were detected (Fig. 4). This may partly be explained by the changes which occur in the expression of cyclin D1, as previously described (15).

Our results also indicate that a decrease in $\beta$-catenin expression is caused by an effect at the protein level rather than at the mRNA level in the activated PKG pathway. Furthermore, a significant increase in the phosphorylation of $\beta$-catenin was detected in the PDE5 siRNA-transfected cells, which was also consistent with previous data indicating that $\mathrm{PKG}$ has a similar role to GSK3 $\beta$ and directly phosphorylates $\beta$-catenin/TCF, leading to proteasomal degradation (16). MG132, an ubiquitinproteasome inhibitor, was used to determine whether the decrease in $\beta$-catenin expression induced by PKG activation could be blocked at the proteasomal level. Our results revealed that MG132 prevented the reduction in $\beta$-catenin expression which was caused by activated PKG, and the inhibitor alone did not alter the level of $\beta$-catenin. These results indicated that the protein was processed by the ubiquitin-proteasome system (Fig. 5A). An increased expression of cyclin D1 levels was also detected compared to the control cells, after MG132 was used to inhibit the ubiquitin-proteasome system (Fig. 6A).

A previous study indicated that JNK activation was required for the PKG-dependent regulation of TCF activity (17). Therefore, in this study, we detected the level of phosphorylation of JNK and found that there was a significant increase in the phosphorylation of JNK in the transfected cells (Fig. 6B). This result is consistent with that of a previous study showing that the activation of PKG directly phosphorylates JNK (18). In addition, there was an increase in the level of cyclin D1 and no 
A

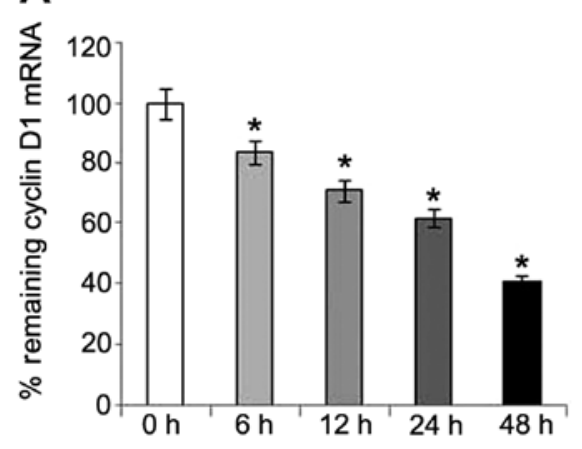

C

$0122448 \mathrm{~h}$

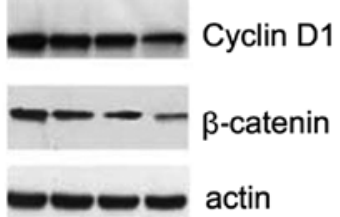

D

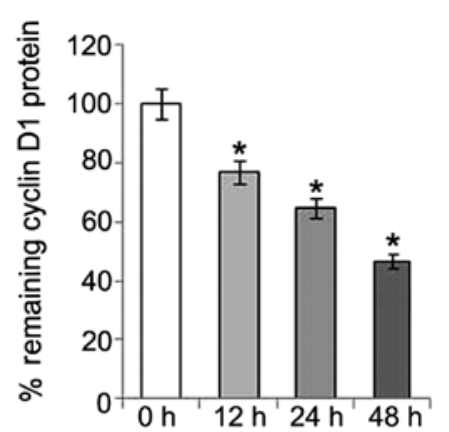

B

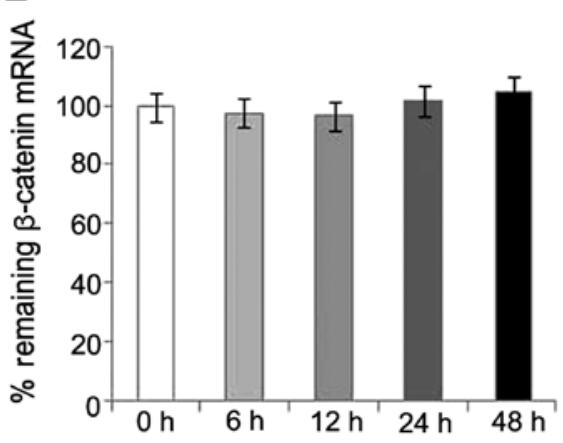

E

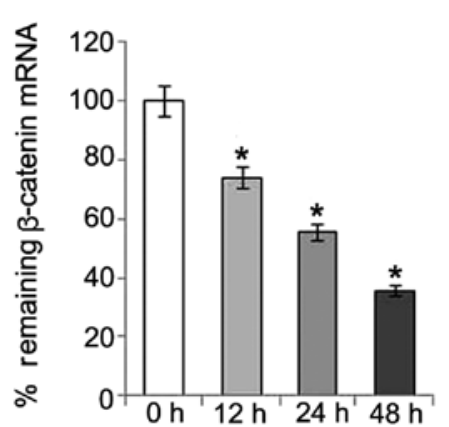

Figure 4. Effects of phosphodiesterase type 5 (PDE5) siRNA on the expression of cyclin D1 and $\beta$-catenin. (A) mRNA expression of cyclin D1 mRNA at different time points. (B) mRNA expression of $\beta$-catenin at different time points. (C) Protein expresssion of cyclin D1 and $\beta$-catenin as shown by western blot analysis. (D) Percentage of cyclin D1 protein expression at different time points. (E) Percentage of $\beta$-catenin protein expression at different time points. Data are presented as the means $\pm \mathrm{SD}(\mathrm{n}=3)$. ${ }^{*} \mathrm{P}<0.05$.

A
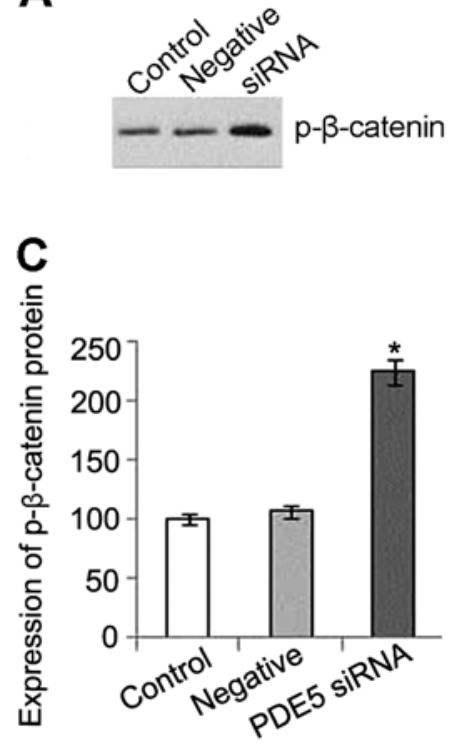

B $\quad-+---$ Negative siRNA

-++-+ PDE5A siRNA

--+++ MG132

$=-\infty-\infty$-catenin

actin

D

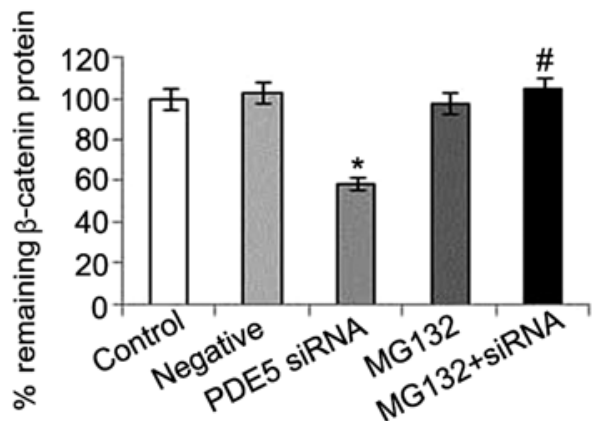

Figure 5. (A) Effect of phosphodiesterase type 5 (PDE5) siRNA on phosphorylated (p)- $\beta$-catenin protein expression. PDE5 siRNA was transfected into the OS-RC-2 cells for $24 \mathrm{~h}$. (B) Expression of $\beta$-catenin in OS-RC-2 cells following treatment with PDE5 siRNA, or MG132, or both. (C) Percentage of p- $\beta$-catenin protein expression. (D) Percentage of $\beta$-catenin protein expression. Data are presented as the means $\pm \mathrm{SD}(\mathrm{n}=3)$. ${ }^{*} \mathrm{P}<0.05$ compared with the control cells. ${ }^{\text {"P }}<0.05$ compared with the PDE5 siRNA group.

change in the levels of $\mathrm{p}$ - $\beta$-catenin following treatment with an inhibitor of JNK, indicating that activated JNK may affect the downstream molecules of $\beta$-catenin/TCF (Fig. 6A).
PKG activation increases the expression of p21 through Spl. To examine whether the activation of PKG alters the transcription of p21, we treated the OS-RC-2 cells with PDE5 siRNA or 

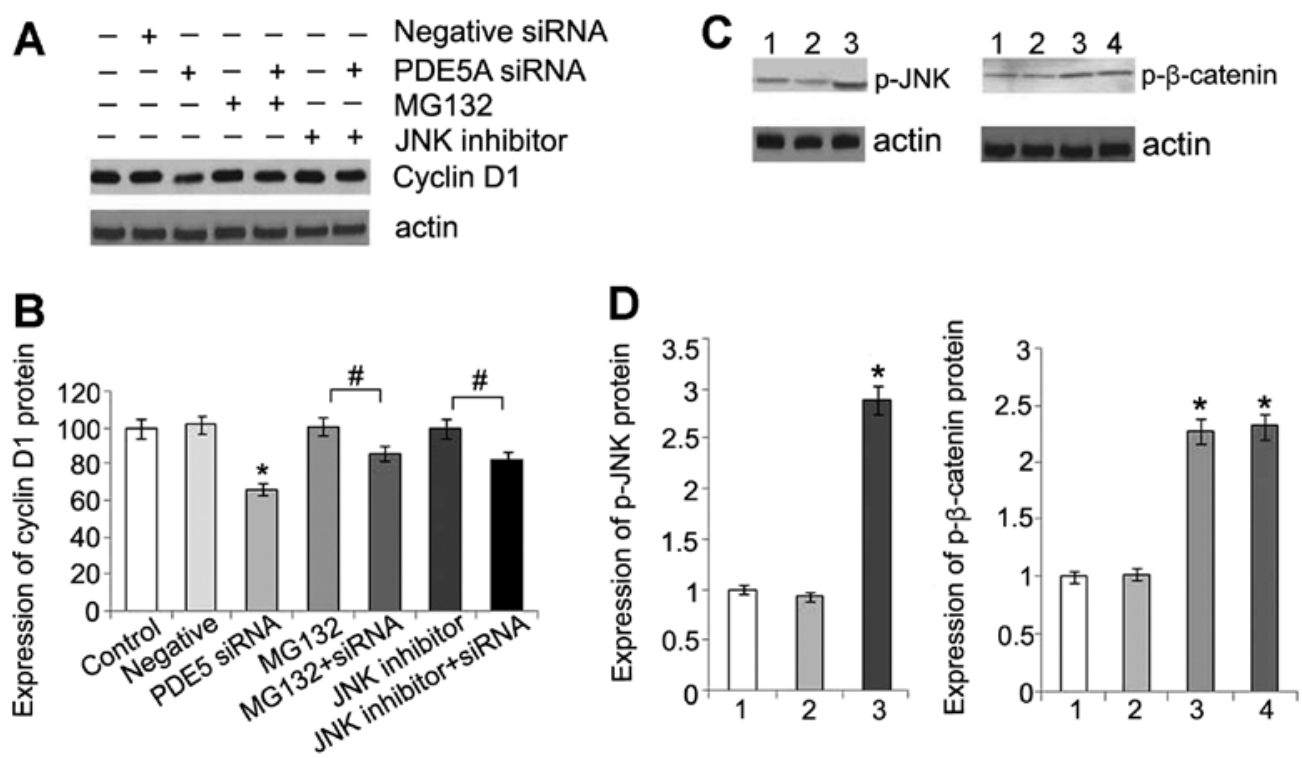

Figure 6. (A) Expression of cyclin D1 protein in OS-RC-2 cells following treatment with phosphodiesterase type 5 (PDE5) siRNA, or MG132, or a combination of both, or c-Jun N-terminal kinase (JNK) inhibitor, or a combination of PDE5 siRNA and JNK inhibitor. (B) Statistical analysis of cyclin D1 protein expression. Data are presented as the means \pm standard deviation (SD) $(n=3)$. ${ }^{*}<<0.05$ compared with control cells. ${ }^{*} \mathrm{P}<0.05$. (C) Comparison of phosphorylated (p)-JNK in OS-RC-2 cells in the different groups by western blot analysis (lane 1, control group; lane 2, negative control group; lane 3, PDE5 siRNA group). Comparison of p- $\beta$-catenin in OS-RC-2 cells in the different groups by western blot analysis (lane 1, control group; lane 2, negative control group; lane 3, PDE5 siRNA group; lane 4, PDE5 siRNA + JNK inhibitor group). (D) Statistical analysis of p-JNK and p- $\beta$-catenin protein. Data are presented as the means \pm SD (n=3). ${ }^{*} \mathrm{P}<0.05$ compared with control.

A
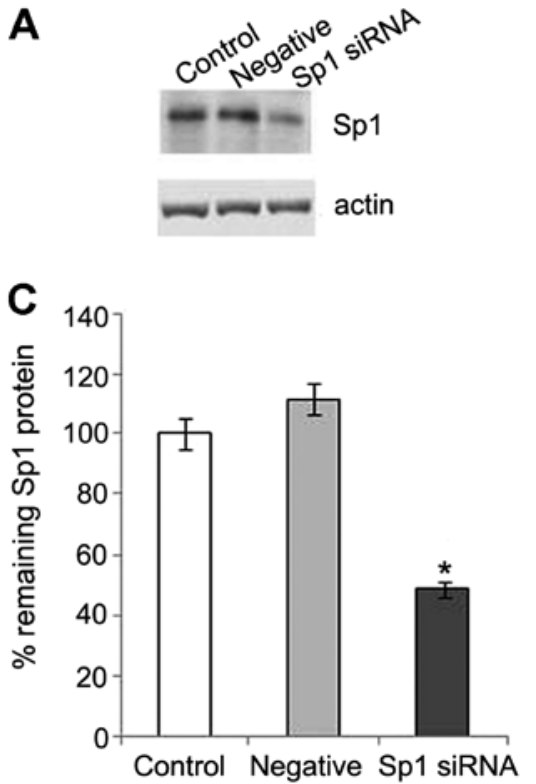

B
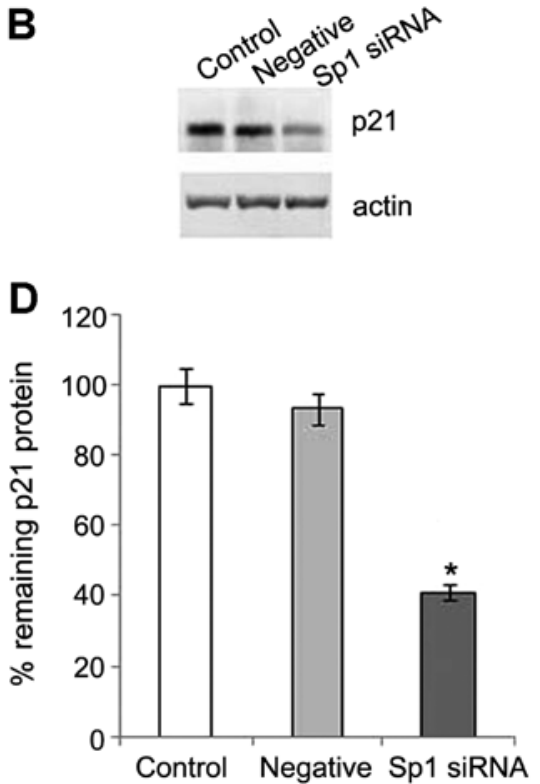

Figure 7. (A) Expression of Sp1 protein in OS-RC-2 cells transfected with Sp1 siRNA. (B) Expression of p21 protein in OS-RC-2 cells transfected with Sp1 siRNA. The cells were pre-treated with 8-Br-cyclic GMP (cGMP) for 24 h. (C) Percentage of Sp1 protein in OS-RC-2 cells transfected with Sp1 siRNA. (D) Percentage of $\mathrm{p} 21$ protein in OS-RC-2 cells transfected with Sp1 siRNA. Data are presented as the means $\pm \mathrm{SD}\left(\mathrm{n}=3\right.$ ). ${ }^{*} \mathrm{P}<0.05$ compared with control group.

8-Br-cGMP. Our results revealed that the mRNA levels of p21 were increased in the cells treated with PDE5 siRNA or 8-BrcGMP; however, the increase was not time-dependent (data not shown). Previous studies have confirmed that $\mathrm{Sp1}$ is required for the PKG-induced upregulation of p21, p27 and HINT1 in SW480 cells, and can be phosphorylated by activating PKG on serine residues $(11,12)$. To further investigate the requirement of $\mathrm{Sp} 1$ in the increase in $\mathrm{p} 21$ expression induced by PKG in
OS-RC-2 cells, we knocked down the expression of endogenous Sp1 by transfecting the cells with Sp1 siRNA. The level of Sp1 in the OS-RC-2 cells was markedly reduced by Sp1 siRNA. The activation of $\mathrm{p} 21$ protein expression was clearly observed in the control group following treatment with 8-BrcGMP, and was inhibited by the suppression of Sp1 (Fig. 7). Thus, our data indicate that the increased expression of $\mathrm{p} 21$ may be regulated through Sp1 by activating PKG. 
A

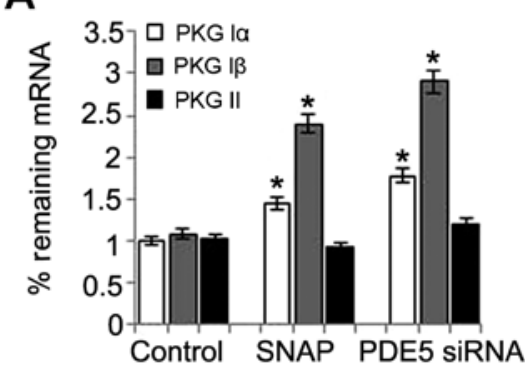

B

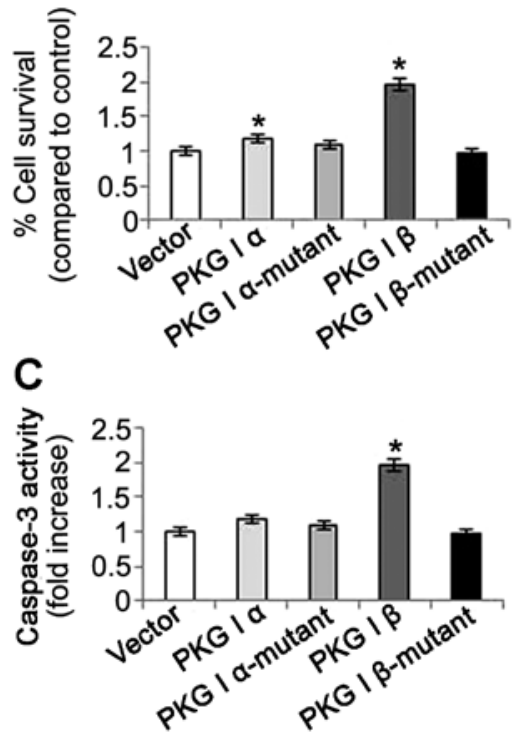

D
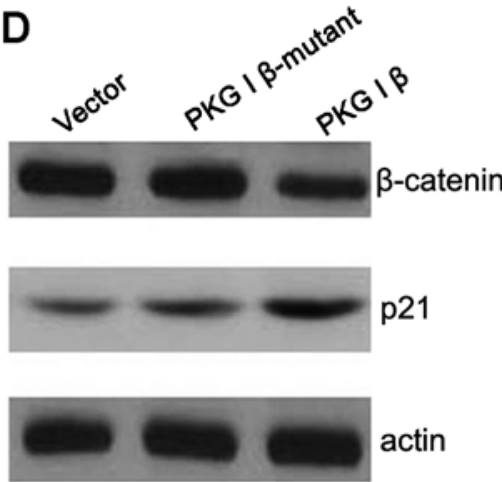

E

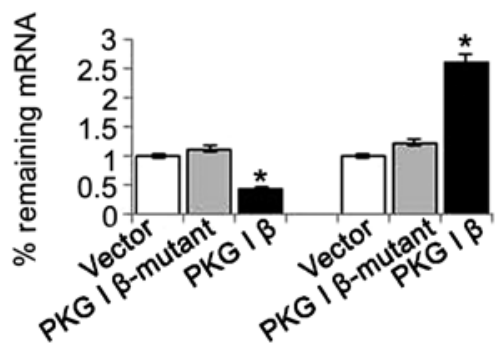

Figure 8. (A) mRNA expression of protein kinase G (PKG) I $\alpha$, I $\beta$ and II in OS-RC-2 cells transfected with phosphodiesterase type 5 (PDE5) siRNA or treated with S-Nitroso-N-acetylpenicillamine (SNAP). (B) Cell proliferation in OS-RC-2 cells treated with PKG I $\alpha$, PKG I $\alpha$-mutant, PKG I $\beta$ and PKG I $\beta$-mutant by MTT assay. (C) Caspase- 3 activity in OS-RC-2 cells treated with PKG I $\alpha$, PKG I $\alpha$-mutant, PKG I $\beta$ and PKG I $\beta$-mutant. (D) The protein expression of $\beta$-catenin and p21 in OS-RC-2 cells treated with PKG I $\beta$ and PKG I $\beta$-mutant by western blot analysis. (E) Statistical analysis of phosphorylated (p)- $\beta$-catenin and p21 protein. Data are presented as the means $\pm \mathrm{SD}(\mathrm{n}=3)$. ${ }^{*} \mathrm{P}<0.05$.

$P K G$ I $\beta$ plays a vital role in anticancer activities in $O S-R C-2$ cells. PKG has various isozymes, including PKG I and PKG II; the $\mathrm{N}$ terminus of PKG $\mathrm{I}$ is encoded by 2 alternatively spliced exons that produce the isoforms $\alpha$ and $\beta$ (19). In this study, we investigate which type of PKG is responsible for the anticancer activities in OS-RC-2 cells. The mRNA expression of PKG I and PKG II was detected in the cells treated with PDE5 siRNA. The results revealed that the mRNA expression of PKG I $\alpha$ and PKG I $\beta$ was significantly increased. The same result was also observed in the nitric oxide (NO) donor SNAP group, which indicated that the NO-cGMP pathway was involved in the anticancer activities induced by the activation of PKG. Unexpectedly, there were no changes observed in the PKG II levels in the cells treated with PDE5 siRNA of SNAP when compared with the control (Fig. 8A). These results suggested that not PKG II, but PKG I was involved in the anticancer activities. Next, we investigated the effects of PKG I $\beta$ overexpression on cell proliferation and apoptosis. The results revealed that the cells with an overexpression of PKG had significantly increased growth rates, whereas this increase was abolished by treatment with the mutants (Fig. 8B). Relative caspase-3 expression was significantly increased in the cells with PKG I $\beta$ overexpression, but there was no obvious change in the cells with PKG I $\alpha$ overexpression (Fig. 8C). However, the role of PKG I was according to its intact structure as no changes in cell proliferation and apoptosis were observed in the cells overexpressing mutant PKG I $\alpha$ and $\mathrm{I} \beta$ (Fig. 8B and $\mathrm{C}$ ). Futhermore, immunoblot analysis revealed that the protein expression of $\beta$-catenin was reduced and the protein expression of p21 was increased in the PKG I $\beta$ group (Fig. 8D). These results suggest that PKG I $\beta$ plays a vital role in anticancer activities in OS-RC-2 cells.

\section{Discussion}

PDE5 inhibition induces sustained levels of intracellular cGMP and activates its downstream PKG, resulting in the inhibition of proliferation and the induction of apoptosis in colon cancer cells $(11,12)$. In this study, we demonstrated that suppressing PDE5 gene expression by PDE5 siRNA transfection inhibited proliferation and induced apoptosis in OS-RC-2 human renal carcinoma cells. The effects were enhanced by 8-Br-cGMP, a cell membrane permeable cGMP derivative, and blocked by KT5823, a PKG inhibitor. Our data support previous findings showing that inhibiting PDE5 by exisulind and its higher affinity analogues, such as CP461 induces apoptosis in colon tumor cells throug the cGMP-PKG signaling pathway $(4,5,12,16)$. The main activated $P K G$ isoform in OS-RC-2 cells is PKG I $\beta$, which is responsible for the anti- 
cancer activities, such as the inhibition of cell proliferation and the increase in the cell apoptotic rate. It has been reported that the activation of soluble guanylyl cyclase by NO leads to an increase in cGMP synthesis and the activation of PKG (20). In this study, the PKG I $\alpha$ and PKG I $\beta$ were significantly increased in the SNAP group, indicating that NO was involved in the cGMP-PKG pathway.

Activated cGMP-PKG may play a key role in determining the levels of cellular $\beta$-catenin that has been well known for its role in carcinogenesis. It has been found that various types of human cancer, such as kidney (21), liver (22), prostate (23) and colon cancer (24) involved an abnormal Wnt signaling pathway characterized by an accumulation of $\beta$-catenin. Moreover, $\beta$-catenin plays a central role in oncogenesis through tge regulation of cell adhesion and the Wnt signaling pathways (25). Along with TCF/Lef, accumulated $\beta$-catenin facilitates the expression of multiple genes involved in proliferation and apoptosis. However, mutations of adenomatous polyposis coli (APC) in tumor cells prevent the formation of the APC/axin/ GSK3 $3 / \mathrm{PP} 2 \mathrm{~A}$ complex, leading to a defective phosphorylation of $\beta$-catenin by GSK3b kinase (26-28). Finally, $\beta$-catenin is unable to be degraded by the ubiquitin-proteasome pathway, resulting in an accumulation of a cellular $\beta$-catenin. We found that the reduction of $\beta$-catenin was followed by an increased phosphorylation of $\beta$-catenin in the OS-RC- 2 cells treated with PDE5 siRNA. Our data confirmed previous findings that the attenuation of cellular $\beta$-catenin accumulation through an activated cGMP-PKG pathway and the increased phosphorylation of $\beta$-catenin occur in exisulind-treated colonic tumor cells. Moreover, it has been suggested that PKG can phosphorylate $\beta$-catenin directly, leading to increased proteasomal degradation in a manner mimicking the effect of GSK3 $\beta$ (16).

The reduction in $\beta$-catenin expression suggested that either an activated proteolysis pathway or an inhibited transcription was initiated in the OS-RC-2 cell transfected with PDE5 siRNA. The levels of $\beta$-catenin mRNA did not decrease within $48 \mathrm{~h}$ following transfection with PDE5 siRNA, indicating an involvement of proteolysis. In this study, we confirmed directly that the levels of $\beta$-catenin could not be reduced by MG132, an ubiquitin-proteasome inhibitor in OS-RC-2 cells transfected with PDE5 siRNA. Furthermore, the proteolysis of $\beta$-catenin was induced not only by the ubiquitin-proteasomal pathway, but also by caspases. A previous study demonstrated that the inhibition of caspase- 3 did not block the degradation of $\beta$-catenin in exisulind-treated cells (29), indicating the necessity of the ubiquitin-proteasome pathway in the proteolysis of $\beta$-catenin. Further studies are required to determine the function of caspases in OS-RC-2 cells transfected with PDE5 siRNA.

$\beta$-catenin/TCF/Lef controls the expression of a number of oncogenes, including cyclin D1, c-myc and peroxisome proliferator-activated receptor (PPAR) $\beta$. The transcription of cyclin D1 can be initiated by the accumulation of $\beta$-catenin $(11,15)$. It has been found that the levels of $\beta$-catenin are related to the activated pathway of cGMP-PKG in various tumor cells treated with exisulind and its higher affinity analogues $(5,12)$. This study demonstrated that $\beta$-catenin protein and both cyclin D1 mRNA and protein levels decreased in a time-dependent manner, indicating that the decrease in $\beta$-catenin levels promoted the downregulation of TCF/Lef-mediated promoter transcription. The suppression of cyclin D1 by antisense oligonucleotide can induce apoptosis in squamous carcinoma cells (30), suggesting that the reduction in cyclin D1 levels may play a direct role in promoting the progression of apoptosis in PDE5 siRNA-transfected cells. However, we found that the reduction in cyclin D1 levels was not blocked by MG132. This may be explained by the fact that cyclin D1 protein can be degraded through an ubiquitin-proteasome pathway triggered by GSK3 $\beta$ phosphorylation. The reduction in cyclin D1 expression can be blocked by an inhibitor of JNK. This supports previous findings that the phosphorylation and activation of the MEKK1-SEK1-JNK1 pathway triggered by either exisulindmediated PKG activation or a constitutively active mutant PKG construct promotes cGMP-mediated apoptosis (18). Therefore, the reduction in cyclin D1 expression is mainly affected by the $\beta$-catenin and JNK pathways, increasing the apoptosis of OS-RC-2 cells.

The results for the levels of p21 and p27 are not similar in different colon cancer cell lines treated with exisulind; i.e., the levels of both were increased in SW480 cells (12) and only the level of p21 protein was increased in HT29 cells (4). However, all the changes were reversed by KT5823, showing that both p21 and p27 are downstream targets of activated PKG. In this study, the increased levels of $\mathrm{p} 21$ protein were blocked by Sp1 siRNA in 8-Br-cGMP-treated OS-RC-2 cells. Therefore, the mechanism involved in the changes of p21 may be related to the activation of Sp1 by PKG in 8-Br-cGMP-treated OS-RC-2 cells. Further study supports the hypothesis that the activation of PKG can lead to the serine phosphorylation of the transcription factor, $\mathrm{Sp}$ (30). In conclusion, decreasing levels of cyclin D1 and increasing levels of $\mathrm{p} 21 \mathrm{can}$ inhibit proliferation and promote apoptosis through the cGMP-PKG pathway in OS-RC-2 cells transfected with PDE5 siRNA.

\section{Acknowledgements}

This study was supported by a grant from the Natural Foundation of Ningbo Science and Technology Bureau, China, (2010A610047). This study also was supported by a grant from the Technology foundation of Yinzhou Science and Technology Bureau, China.

\section{References}

1. Rini BI, Rathmell WK and Godley P: Renal cell carcinoma. Curr Opin Oncol 20: 300-306, 2008.

2. Escudier B: Chemo-immunotherapy in RCC: the end of a story. Lancet 375: 613-614, 2010.

3. Mulders PF, Brouwers AH, Hulsbergen-van der Kaa CA, et al: Guideline 'Renal cell carcinoma'. Ned Tijdschr Geneeskd 152: 376-380, 2008 (In Dutch).

4. Zhu B and Strada SJ: The novel functions of cGMP-specific phosphodiesterase 5 and its inhibitors in carcinoma cells and pulmonary/cardiovascular vessels. Curr Top Med Chem 7: 437-454, 2007.

5. Thompson WJ, Piazza GA, Li H, et al: Exisulind induction of apoptosis involves guanosine 3',5'-cyclic monophosphate phosphodiesterase inhibition, protein kinase $\mathrm{G}$ activation, and attenuated beta-catenin. Cancer Res 60: 3338-3342, 2000.

6. Piazza GA, Thompson WJ, Pamukcu R, et al: Exisulind, a novel proapoptotic drug, inhibits rat urinary bladder tumorigenesis. Cancer Res 61: 3961-3968, 2001.

7. Zhu B, Vemavarapu L, Thompson WJ and Strada SJ: Suppression of cyclic GMP-specific phosphodiesterase 5 promotes apoptosis and inhibits growth in HT29 cells. J Cell Biochem 94: 336-350, 2005. 
8. Lim JT, Piazza GA, Han EK, et al: Sulindac derivatives inhibit growth and induce apoptosis in human prostate cancer cell lines. Biochem Pharmacol 58: 1097-1107, 1999.

9. Tinsley HN, Gary BD, Keeton AB, et al: Sulindac sulfide selectively inhibits growth and induces apoptosis of human breas tumor cells by phosphodiesterase 5 inhibition, elevation of cyclic GMP, and activation of protein kinase G. Mol Cancer Ther 8 : 3331-3340, 2009

10. Tinsley HN, Gary BD, Keeton AB, et al: Inhibition of PDE5 by sulindac sulfide selectively induces apoptosis and attenuates oncogenic Wnt/beta-catenin-mediated transcription in human breast tumor cells. Cancer Prev Res (Phila) 4: 1275-1284, 2011.

11. Tetsu $\mathrm{O}$ and McCormick F: Beta-catenin regulates expression of cyclin D1 in colon carcinoma cells. Nature 398: 422-426, 1999.

12. Li H, Liu L, David ML, et al: Pro-apoptotic actions of exisulind and CP461 in SW480 colon tumor cells involve beta-catenin and cyclin D1 downregulation. Biochem Pharmacol 64: 1325-1336, 2002.

13. Livak KJ and Schmittgen TD: Analysis of relative gene expression data using real-time quantitative PCR and the 2(-Delta Delta C(T)) method. Methods 25: 402-408, 2001.

14. Deguchi A, Thompson WJ and Weinstein IB: Activation of protein kinase $\mathrm{G}$ is sufficient to induce apoptosis and inhibit cell migration in colon cancer cells. Cancer Res 64: 3966-3973, 2004

15. Carlson B, Lahusen T, Singh S, et al: Downregulation of cyclin D1 by transcriptional repression in MCF-7 human breast carcinoma cells induced by flavopiridol. Cancer Res 59: 4634-4641, 1999.

16. Liu L, Li H, Underwood T, et al: Cyclic GMP-dependent protein kinase activation and induction by exisulind and CP461 in colon tumor cells. J Pharmacol Exp Ther 299: 583-592, 2001.

17. Kwon IK, Wang R, Thangaraju M, et al: PKG inhibits TCF signaling in colon cancer cells by blocking beta-catenin expression and activating FOXO4. Oncogene 29: 3423-3434, 2010.

18. Soh JW, Mao Y, Kim MG, et al: Cyclic GMP mediates apoptosis induced by sulindac derivatives via activation of c-Jun NH2-terminal kinase 1. Clin Cancer Res 6: 4136-4141, 2000.

19. Hofmann F: The biology of cyclic GMP-dependent protein kinases. J Biol Chem 280: 1-4, 2005.
20. Das A, Xi L and Kukreja RC: Protein kinase G-dependent cardioprotective mechanism of phosphodiesterase-5 inhibition involves phosphorylation of ERK and GSK3beta. J Biol Chem 283 29572-29585, 2008.

21. Peruzzi B and Bottaro DP: Beta-catenin signaling: linking renal cell carcinoma and polycystic kidney disease. Cell Cycle 5: 2839-2841, 2006.

22. Thompson MD and Monga SP: WNT/beta-catenin signaling in liver health and disease. Hepatology 45: 1298-1305, 2007.

23. Chesire DR and Isaacs WB: Beta-catenin signaling in prostate cancer: an early perspective. Endocr Relat Cancer 10: 537-560, 2003.

24. Stein U, Arlt F, Smith J, et al: Intervening in beta-catenin signaling by sulindac inhibits S100A4-dependent colon cancer metastasis. Neoplasia 13: 131-144, 2011.

25. Li H, Pamukcu R and Thompson WJ: beta-Catenin signaling: therapeutic strategies in oncology. Cancer Biol Ther 1: 621-625, 2002.

26. Ueda M, Gemmill RM, West J, et al: Mutations of the beta-and gamma-catenin genes are uncommon in human lung, breast, kidney, cervical and ovarian carcinomas. Br J Cancer 85: 64-68, 2001.

27. Tarmin L, Yin J, Harpaz N, et al: Adenomatous polyposis coli gene mutations in ulcerative colitis-associated dysplasias and cancers versus sporadic colon neoplasms. Cancer Res 55: 2035-2038, 1995.

28. Lewis A, Davis H, Deheragoda M, et al: The C-terminus of Apc does not influence intestinal adenoma development or progression. J Pathol 226: 73-83, 2012.

29. Whitehead CM, Earle KA, Fetter J, et al: Exisulind-induced apoptosis in a non-small cell lung cancer orthotopic lung tumor model augments docetaxel treatment and contributes to increased survival. Mol Cancer Ther 2: 479-488, 2003.

30. Saikawa Y, Kubota T, Otani Y, et al: Cyclin D1 antisense oligonucleotide inhibits cell growth stimulated by epidermal growth factor and induces apoptosis of gastric cancer cells. Jpn J Cancer Res 92: 1102-1109, 2001. 